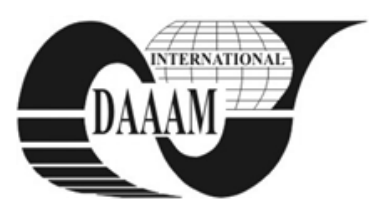

Annals of DAAAM for 2011 \& Proceedings of the 22nd International DAAAM Symposium, Volume 22, No. 1, ISSN 1726-9679 ISBN 978-3-901509-83-4, Editor B. Katalinic, Published by DAAAM International, Vienna, Austria, EU, 2011 Make Harmony between Technology and Nature, and Your Mind will Fly Free as a Bird Annals \& Proceedings of DAAAM International 2011

\title{
COMPLEX DEVICE FOR WATER ANALYTIC
}

\section{GUTT, G[heorghe]; GUTT, S[onia]; POROCH - SERITAN, M[aria] \& GUTT, A[ndrei]}

\begin{abstract}
Complex photometer described in the paper is a portable device with which it is possible to determine simultaneously both the concentration of certain chemical species in water as well as its electrolytic conductivity. The device can be used for the analysis of independent samples that are found in glass tubes, or of water in displacement through the flow cells using for measurements the flow injection analysis (FIA) (Flow Injection Analysis).
\end{abstract}

Key words: photometer, conductometer, flow cell

\section{INTRODUCTION}

The nature of chemical species in water and their concentration can be determined only by photocolorimetric optical absorbance value after in analysed water were caused specific color reactions in as many water samples as chemical species of interest. Considering that water is colorless, for the purposes of photometric analysis of water samples it is necessary in the first phase to be completed a color reaction between followed chemical species and a specific reactant, resulted color intensity being proportional to the concentration of chemical species pursued. In the second phase, the resulted colored solution is photometrically analyzed at a wavelength corresponding to followed species with the aim of establishing the optical absorbance of the solution and absorbance value is correlated with concentration through the Beer Lambert Law (Matter, 1995, Skoog,1996, Robinson,2005). Currently, in the photometry of water are used in a large-scale pre-measured dye kits for a given volume of water so that photometric analysis (Gutt S., 2010-1; 2010-2; 2010-3) of water can be performed in situ using portable photometers (Gutt S., 2010).

Measurement of electrolytic conductivity of water is used to determine the cumulative effect of cations and anions concentration in water Electrolytic conductivity value is not selective in terms of indicating the presence of a certain chemical species, (Gutt G., 2010) and in determination of its concentration, it provides only an overview, but very important, its contamination.

\section{EXPERIMENTAL}

To determine the concentration of various chemical species in water calibrated photometers at particular wavelength are used, determining a single chemical species, or programmable spectrophotometers with monochromator which can provide a wide range of wavelength values, each of them being specific to a particular chemical species. For the work in the visible spectral analysis both in photometric and spectrophotometric analyzes a specific color reaction is required for each species.

In order to determine the electrolytic conductivity cell contact or contactless devices are used. In the case of cell contact device analyzed water is in contact with two plane parallel platinum electrodes, with a certain area, located at a precise distance apart and electrolytic conductivity is determined by the value of the electrolyte ohmic resistance of the electrolyte column between the two electrodes. In the case of devices with contactless conductivity cells, the electrodes are located on the outer wall of the cell, they were the armatures of a capacitor that is part of an oscillating circuit whose frequency is an expression of the dielectric conductivity (analyzed water). The solution presented by the authors in the paper refers to a modular and combined portable device to determine in situ and simultaneous both the concentration of a chemical species in water, by photometric way as well as the electrolytic conductivity of water using the principle of contactless conductivity cell. The equipment produced by the authors is extremely simple being composed of a clamp, type photobarrier that can be fixed by elastic grip with a spring on a glass tube, containing water to be analyzed in static conditions either in a glass tube through which water flows in bypass mode, pumped from a pool of water, in the latter case it is used the flow analysis system (FIA). The basic structure of equipment is composed of a photometric clamp, figure 1, which has two semicircular electrodes of stainless steel sheet on each clamping die molded on glass tube, each electrode presenting a hole through which the radiation of a LED, passes on a clamping die of the clip, to a photodiode receiver, located on the other clamping die, monochromatic radiation emitted by the LED having a wavelength corresponding to maximum optical absorbance of the analyzed chemical species, the combined

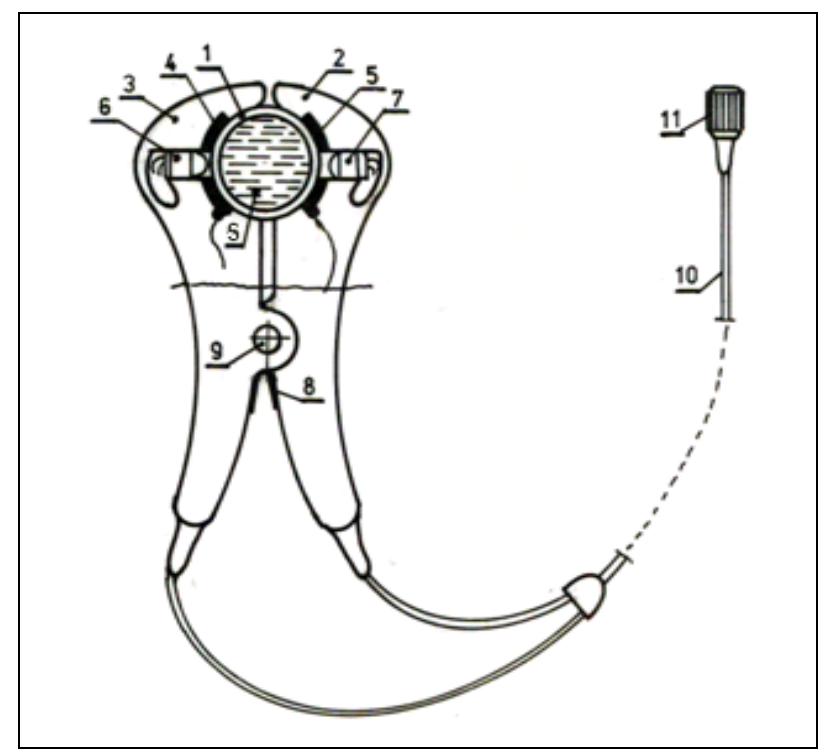

Fig. 1. View with section of the clamp of the portable combined photometer. 1-glass tube with analyzed solution, 2, 3-clamping dies, 4, 5-electrodes, 6-LED emitter, 7-photodiode receiver, 8spring for elastic grip, 9-pin, 10-electrical cable, 11-electrical connector, S-test solution 


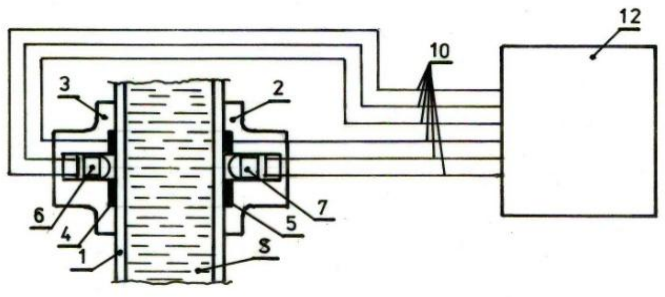

Fig. 2. Principle scheme of portable combined photometer: 1-glass - tube with analyzed solution, 2, 3-clamping dies, 4, 5electrodes, 6 - LED emitter, 7-photodiode receiver, 10-electric cable, 12-electronic unit, $\mathrm{S}$-test solution

photometer also includes an electrical cable for connection and an electronic part. Operations for electrolytic conductivity and concentration measurement with device described involve catching of conductivity sensor on the cylindrical glass tube pushing the clamp arms with two fingers, positioning the clamp on the tube in the desired position followed by weakness of pressure on the arms and reading the value of chemical species concentration and the total electrolyte conductivity on the alphanumeric display, figure 2, of the electronic unit.

\section{RESULTS AND DISCUSSION}

Electrolytic conductivity is first determined using the combined device for water analytic, followed by realization of color reaction and photometry of colored solution to determine the concentration of pursued species. Electrolytic conductivity is determined by the change in condenser capacity consists of two planar electrodes 4 and 5 outer of glass tube 1 due to changes in dielectric properties (tested water column) located between its armatures (the two external electrodes), capacity having the following expression:

$$
C=\varepsilon_{0} \varepsilon_{r} \cdot \frac{A}{d}
$$

where:

$C$ - condenser capacity with plan armatures;

$\varepsilon_{0}$ - absolute power permeability in vacuum;

$\varepsilon_{r}$ - permeability of water column between electrodes;

$A$ - surface area of external electrodes;

$d$ - distance between electrodes;

Since direct measurement of the capacitor capacity due to permeability changes of water column between the electrodes, permeability that express the electrical conductivity of water is quite difficult and not provide a sufficient resolution and accuracy, capacitor is connected in an $L C$ oscillator circuit whose oscillation frequency $f_{0}$ is the expression:

$$
f_{O}=\frac{1}{2 \pi \sqrt{L C}}
$$

Any change in the ionic composition of water between the armatures determines a different value of relative electric permeability, value which in turn causes a deviation $\Delta f$ of the frequency oscillation $f$ from the resonance frequency $f_{0}$ :

$$
\Delta f=f-f_{0}
$$

where:

$L$ - inductance coil;

$C$ - condenser capacity.

Therefore, oscillatory circuit frequency deviation expressed in terms of a constant surface of electrodes and a constant distance between them, electrical conductivity of water samples analyzed:

$$
\chi=k \cdot \Delta f
$$

which can be automatically determined based on a standard curve, in coordinates: electrolytic conductivity $(\chi)$ - frequency deviation $(\Delta f)$, stored electronically, by extrapolating of measured values of frequency deviation $\Delta f$ on the curve.

The basis for determining the concentration of followed species is Lambert-Beer law which establishes the connection between the optical absorbance $A$ of the colored solution, thickness of the photometrical layer $b$, molar absorption coefficient $a$ and the concentration $c$ of the species observed:

$$
A=a \cdot b \cdot c
$$

In the given conditions, sizes a and $\mathrm{b}$ have constant values, such as equation (1) can be expressed as:

$$
A=k \cdot c
$$

last relationship representing the equation of the calibration curve.In order to achieve the calibration curve photometric absorbances $A_{1} \ldots A_{n}$ of colored solutions are measured in turn, solutions having concentrations of the species pursued $c_{1 \ldots . . .} c_{n}$, calibration solutions were made in turn by pure analytical reagents.

After photometric analysis of all standard pairs of values, photometrical absorbance $A$-concentrations $c$ are stored into computer memory.

Any subsequent measurement of photocolorimetrical absorbance of a sample is automatically extrapolated by software on this curve, and the value of concentration as a result of extrapolation is displayed.

\section{CONCLUSION}

Upon conclusion of the exposed concept are established the foundations of a combined portable, simple device, low cost price and requiring no maintenance, precision and high productivity, both of electrolytic conductivity of water as well as concentration of a certain chemical species of water.

The determination of each other chemical species needs another photometric clip with a radiation source type LED which has the emission wavelength value equal to the wavelength of radiation absorbed by that specific species .

\section{REFERENCES}

Gutt, S. (2007-1). Photometric probe, (Romanian), Patent RO $122.598 / 2007$

Gutt, S. (2007-2). Miniature Spectrometer (Romanian), Patent RO $122.600 / 2007$

Gutt, S.; Gutt, G. \& Winkler, I.. (2010). Contributions to the development of photometric and spectrophotometric portable kits for water analysis, Simpozion NATO, Advanced Reserch Workshop, Lviv., 19-22.05.2010, Springer Netherlands, pp. 10

Matter, L.. (1995). Lebensmittel- und Umweltanalytik mit der Spektrometrie, VCH Verlagsgesellschaft, Weinheim, pp. $111-131$

Skoog D.A, Leary J.J. .- Instrumentelle Analytik, Grundlagen Geräte, Anwendungen, Springer Verlag, Berlin Heidelberg, 1996, pp.108-123

Robinson J.W., Skelly Frame E., M., Frame II G.,M. Undergraduate Instrumental Analysis, Marcel Decker, New York , 2005, pp.83-92 